\title{
Effective Delivery of PEGylated siRNA-Containing Lipoplexes to Extraperitoneal Tumours following Intraperitoneal Administration
}

\author{
Akul Singhania, Sherry Y. Wu, and Nigel A. J. McMillan \\ The University of Queensland Diamantina Institute, Princess Alexandra Hospital, The University of Queensland, Woolloongabba, \\ QLD 4102, Australia \\ Correspondence should be addressed to Nigel A. J. McMillan, n.mcmillan@uq.edu.au \\ Received 14 January 2011; Accepted 7 April 2011 \\ Academic Editor: Hsin-I Chang \\ Copyright () 2011 Akul Singhania et al. This is an open access article distributed under the Creative Commons Attribution License, \\ which permits unrestricted use, distribution, and reproduction in any medium, provided the original work is properly cited. \\ Intraperitoneal (i.p.) administration of small interfering RNA (siRNA) has, to date, shown promise in treating tumours located \\ within the peritoneal cavity. The ability of these siRNA molecules to reach extraperitoneal tumours following i.p. administration \\ is, however, yet to be investigated. Here, we examined the impact of PEGylation on the biodistribution of i.p. administered \\ nucleic acids-containing lipoplexes. We showed that in contrast to non-PEGylated liposomes, PEGylated liposomes can deliver \\ siRNA efficiently to extraperitoneal tumours following i.p. administration, resulting in a $45 \%$ reduction in tumour size when the \\ oncogene-targeted siRNA was used. This difference was likely contributed by the decreased uptake of PEGylated lipoplexes in \\ the first-pass organs, and, in particular, we observed a 10-fold decrease in the macrophage uptake of these particles compared to \\ non-PEGylated counterparts. Overall, our results indicated the potential of using PEGylated liposomes to deliver siRNA for the \\ treatment of i.p. localized cancer with coexisting extraperitoneal metastasis.
}

\section{Introduction}

Since its discovery, small interfering RNA (siRNA) has been widely investigated as a therapeutic agent to treat a wide range of human diseases, from cancer [1] to infectious [2] or neurodegenerative diseases [3]. For cancer therapy, it is typically administered locally (intracerebral/intratumoral injections) or systemically (intravenous injections) with the use of suitable delivery carriers, such as liposomes or polymers (reviewed in [4]). These carriers often carry a positive charge to interact efficiently with negatively charged siRNA thereby achieving effective entrapment or protection as well as permitting efficient cell entry. The promise of using siRNA to treat cancer has been recently demonstrated in a phase I clinical trial in which the repeated intravenous (i.v.) administrations of siRNA-containing cyclodextrin nanoparticles resulted in a significant downregulation of the targeted M2 subunit of ribonucleotide reductase (RRM2) protein in solid tumours [5].
Despite the success, the common adverse effects of repeated i.v. administration, such as phlebitis or loss of veins [6], along with the risk of embolism following injection [7], warrants research into alternative route of administration for cancer treatments. This is of particular interest for the administration of nucleic acids as the presence of both positively and negatively charged components in the formulation system often results in formation of aggregates in highly concentrated samples [8]. Given the intraperitoneal (i.p.) nature of several cancer types, including cancers of the digestive system, peritoneum, and ovaries [9], the i.p. route of administration presents as an attractive alternative. To date, three randomised phase III trials have already demonstrated the survival benefit of i.p. versus i.v. chemotherapy $[10,11]$ or when adjunct i.p. chemotherapy was used [12] for the treatment of advanced, low-volume ovarian cancer. Importantly, i.p., in contrast to i.v., delivery route permits large volume administration such that formulations can be prepared at dilute concentrations in order to avoid particle aggregation. 
To date, several studies have described the successful i.p. delivery of DNA, antisense, or siRNA, using viral vectors [13, 14], nanoparticles [15], liposomes [1], or polymers [16]. Fewell and colleagues, for example, administered a polymer-complexed anticancer cytokine interleukin-12 gene intraperitoneally into a mouse model of disseminated ovarian cancer, resulting in a significant decrease in vascular endothelial growth factor in tumours and improved survival [16]. Similarly, Landen and colleagues demonstrated a 48$81 \%$ tumour reduction following intraperitoneal administration of liposome-entrapped siRNA and paclitaxel, a chemotherapeutic agent, in a mouse model of ovarian cancer [1]. Importantly, it was reported that the level of tumour reduction observed was comparable to concurrently-treated mice with paclitaxel and siRNA administered via i.v. route [1]. While these studies demonstrated the feasibility of delivering nucleic acids intraperitoneally for treatment of i.p. localised tumour, the potential of these i.p. administered nucleic acids to reach extraperitoneal tumours is yet to be confirmed. This is important as many types of cancers have the tendency to metastasize to extraperitoneal sites with the primary tumour located within the peritoneal cavity $[17,18]$.

To our knowledge, only one study, to date, has reported the potential of delivering siRNA to extraperitoneal tumours following i.p. administration using TransMessenger, a commercially available transfection reagent [19]. The level of tumour delivery as well as factors which could influence the practicality of this approach, however, is still yet to be investigated. The aim of this study was therefore to systematically investigate the potential of siRNA to reach extraperitoneal tumours following i.p. administration. The siRNA molecules was entrapped within either non-PEGylated or PEGylated liposomes and the biodistribution along with the level of uptake by i.p. macrophages were examined. The delivery efficacy of siRNA to extraperitoneal tumours was further assessed in mice bearing E6/7 oncogene-expressed extraperitoneal tumours. Overall, our results indicated the potential of PEGylated liposomes to deliver siRNA to extraperitoneal tumours following i.p. administration and could therefore be of use for the treatment of i.p. localized cancer with coexisting extraperitoneal metastasis.

\section{Materials and Methods}

2.1. Materials. Dioleoyl trimethylammonium propane (DOTAP) and cholesterol were purchased from Sigma (St Louis, Mo, USA). Polyethylene Glycol (PEG) 2000 -C16Ceramide conjugate was from Avanti Polar Lipids (Alabaster, Ala, USA) and dioleoylphosphatidylethanolamine (DOPE) was from Northern Lipids (Vancouver, Canada).

Oligodeoxynucleotides with sense sequence of $5^{\prime}$-GTCAGAAATAGAAACTGGTCATC- $3^{\prime}$ and antisense sequence of 5'-GATGACCAGTTTCTATTTCTGAC-3' were obtained from Invitrogen (Carlbad, Calif, USA). HPV16 E6/7-targeted siRNA (5'-GCAACAGUUACUGCGACGUUU-3'; $5^{\prime}$ ACGUCGCAGUAACUGUUGCUU- $\left.3^{\prime}\right)$ and control siRNA (5'-UUAUGCCGAUCGCGUCACAUU-3'; 5' -UGUGACGCGAUCGGCAUAAUU-3') were purchased from SigmaAldrich (St Louis, Mo, USA) in annealed form.
TC-1 cells (murine C57B/6 lung epithelial cells) were obtained from TC Wu [20] and were cultured in Dulbecco's Modified Eagle Media (DMEM; Invitrogen, Carlsbad, Calif, USA) supplemented with $10 \%$ heat-inactivated fetal bovine serum (FBS; Bovoge, Keilor East, Australia), 0.2\% primocin (InvivoGen, San Diego, Calif, USA), and $2 \mathrm{mM}$ L-glutamine (Invitrogen).

\subsection{Liposome Formulations. Non-PEGylated DOTAP/Cho-} lesterol (1:1 molar ratio) was prepared using the hydration of lipid film method as previously described [21]. Dried lipid film was hydrated using sterile 5\% dextrose solution, and the final liposome concentration was $5 \mathrm{mM}$. After stabilizing at room temperature for two hours, small unilamellar liposomes were obtained via extrusion through $0.1 \mu \mathrm{m}$ pore size Nucleopore track-etched membranes using a Lipex extruder (Northern lipids, Vancouver, Canada). The resultant liposomes were then complexed with oligonucleotides at a nitrogen:phosphate $(\mathrm{N}: \mathrm{P})$ ratio of $4: 1$ with the final oligonucleotides concentration being $24 \mu \mathrm{g} / 300 \mu \mathrm{L}$.

PEGylated lipoplexes were formulated using the hydration of freeze-dried matrix (HFDM) technique [22]. DOTAP, cholesterol, DOPE and PEG $_{2000}-\mathrm{C} 16$ Ceramide with a molar ratio of $50: 35: 5: 10$ were used. Freeze-dried matrix was hydrated with sterile water and the final product contained $24 \mu \mathrm{g}$ oligonucleotides or siRNA in $300 \mu \mathrm{L}$ of isotonic sucrose solution.

The particle size and nucleic acids entrapment efficiency of both formulations were examined using the procedures described previously in Wu et al. [22].

2.3. Animal Studies. All animal experiments were approved by The University of Queensland Animal Ethics Committee, and 2-month-old female C57B/6 mice (Perth, ARC) were used in all studies.

2.3.1. Peritoneal Macrophages Uptake Study. Two separate injections of PEGylated or non-PEGylated lipoplexes containing $36 \mu \mathrm{g}$ of FITC-conjugated oligonucleotides $(450 \mu \mathrm{L} /$ dose $)$ were administered intraperitoneally into each mouse. Injections were performed at the left and right lower quadrant of the peritoneal cavity, and all mice received a total dose of $72 \mu \mathrm{g}$ of liposome-entrapped oligonucleotides or the corresponding amount of empty liposomes. At 6 hours after i.p. administration, euthanized mice were injected intraperitoneally with $5 \mathrm{~mL}$ of PBS-Heparin-FCS ( $5 \%$ heat inactivated FCS and $4 \mathrm{U} / \mathrm{mL} \mathrm{Na}$ heparin, DBL, Hospira Pty Ltd, Lake Forest, Ill, USA). Pooled peritoneal fluid of up to 3 mice was collected for each treatment group, and i.p. macrophages were identified via staining with APC-CD11b (BioLegend, San Diego, CA) and PE-F4/80 (BioLegend) antibodies. The percentages of FITC-positive cells were determined in CD11b- and F4/80-positive population using flow cytometry (BD-FACS Canto).

2.3.2. Biodistribution Study. Mice were inoculated with $1 \times$ $10^{6}$ TC- 1 cells suspended in $100 \mu \mathrm{L}$ PBS subcutaneously at the right abdominal side. On day 14 after the inoculation, 
non-PEGylated or PEGylated lipoplexes, which contained $72 \mu \mathrm{g}$ of Alexa Fluor 750-conjugated oligonucleotides, were injected intraperitoneally using the procedure described above. Empty liposome-treated mice were used as controls. At $24 \mathrm{hrs}$ post injection, tumours and major first-pass internal organs (liver, kidneys, and spleen) were dissected and the fluorescence intensity of each organ was examined using excitation and emission wavelengths of $720 \mathrm{~nm}$ and $790 \mathrm{~nm}$, respectively, in a Kodak In Vivo Imager. Results were subsequently analysed using the Kodak molecular imaging software.

2.3.3. Tumour Growth Inhibition Study. Mice were injected with one million TC- 1 cells, suspended in $100 \mu \mathrm{L}$ of sterile PBS, at the right abdominal side. On day 3, 7, and 10 following tumour cell inoculation, mice were treated intraperitoneally with isotonic sucrose solution (vehicle), empty liposomes, or PEGylated lipoplexes containing $72 \mu \mathrm{g}$ of either E6/7-targeted siRNA or control siRNA. Five mice were used per treatment group, and tumour size was monitored using callipers during the course of the experiment. All mice were sacrificed on day 13, and the tumour size of each mouse was recorded and analysed using the GraphPad Prism software (GraphPad software, La Jolla, CA). Student $t$-test was performed to assess the difference between treatment and control groups.

\section{Results and Discussion}

In order to examine the feasibility of delivering nucleic acids to extraperitoneal tumours following i.p. administration, we first sought to evaluate the level of uptake of oligonucleotides-containing lipoplexes by i.p. macrophages as they serve as our body's first line of defence by efficiently engulfing foreign particles. It was anticipated that a strong uptake of these particles by macrophages present in the peritoneal cavity would likely to significantly hinder their delivery to tumours, similar to what has been established with i.v. route of administration (reviewed in [23]). To address this, we formulated PEGylated lipoplexes in which the presence of PEG polymer on the particle surface would aid in their escape from the immune surveillance [24]. These particles were formulated using the hydration of freeze-dried matrix technique, resulting in final particles with an average size of 180 $200 \mathrm{~nm}$ and an entrapment efficiency of $>90 \%$ [22]. Similar particle size and entrapment efficiency were obtained for non-PEGylated lipoplexes. Oligonucleotides incorporated in these lipoplexes were FITC-labelled such that the level of uptake of these particles by i.p. macrophages could be easily assessed. Importantly, contrary to the study performed by Niu and colleagues where only $12 \mu \mathrm{L}$ of siRNA-containing complexes was administered intraperitoneally into each mouse [19], we administered diluted lipoplexes in a large volume to ensure even distribution of the particles within the peritoneal cavity and to reduce the potential formation of aggregates. This high-volume administration of diluted samples has been previously reported to result in reduced clearance leading to superior efficacy [25-29]. At 6 hrs after i.p. administration of lipoplexes, i.p. macrophages were isolated and identified via CD11b and F4/80 antibodies staining. Based on the FITC fluorescence signal detected in these macrophages, it was estimated that there was a 10 -fold decrease in the level of uptake by macrophages for PEGylated lipoplexes compared to non-PEGylated ones $(6.3 \%$ versus $62.3 \%$, Figure 1 ).

To examine whether this difference in macrophage uptake alters the biodistribution and the delivery efficiency of these particles to extraperitoneal tumours, we next labelled oligonucleotides with an infra red fluorescent dye, Alexa Fluor 750. The biodistribution of both PEGylated and nonPEGylated lipoplexes was subsequently examined in mice bearing extraperitoneal E6/7-expressing tumours. At $24 \mathrm{hrs}$ after administration, it was found that the non-PEGylated lipoplexes accumulated mainly in the liver, spleen, and kidneys while very little tumour accumulation of these particles was observed (7.5\% of total dose remained, Figure 2 and Table 1). In contrast, while a considerable amount of oligonucleotides still accumulated in first-pass organs, as expected $[1,29]$, the delivery of PEGylated lipoplexes to the extraperitoneal tumour was evident (Figure 3). This level of tumour localisation of PEGylated lipoplexes was found to be significantly more than that achieved by non-PEGylated ones $(65.9 \%$ versus $7.5 \%$, Figures 2, 3, and Table 1) and is consistent with what we have previously observed with i.v. route of administration of these particles [8]. Minimal delivery of either non-PEGylated or PEGylated lipoplexes was observed in the lungs (data not shown).

It must be noted that the PEGylated lipoplexes exhibited a significant decrease in spleen uptake compared to nonPEGylated lipoplexes following i.p. administration (7.8\% versus $30 \%$, Table 1 ). This could be contributed by the decreased uptake of these particles by i.p macrophages as it has been shown that intraperitoneal macrophages typically travel through the subcapsular sinus of parathymic lymph nodes and eventually reside in the parenchyma of the liver and spleen [30]. It is therefore likely that, following escape from first-pass organs, PEGylated lipoplexes were absorbed into subdiaphragmatic lymphatics at a much higher rate compared to non-PEGylated particles prior to entering the general circulation, which in turn contributed to their enhanced tumour localisation [6]. In addition, following absorption via mesenteric lymphatics, the slight reduction in liver uptake observed for PEGylated lipoplexes could also have contributed to the increased drainage of the administrative material into the portal veins [31] (Table 1). It is therefore anticipated that the PEGylated lipoplexes reached the extraperitoneal tumours via a combination of portal and lymphatics pathways. In contrast, diffusion across the peritoneum is less likely to be the route of transport for these particles to extraperitoneal sites as previous reports have shown that the largest pore size in the peritoneum is less than $40 \mathrm{~nm}[32,33]$, which is significantly smaller than the size of our lipoplexes.

Having established the ability of PEGylated liposomes in delivering oligonucleotides to extraperitoneal tumours following i.p. administration, we next performed an efficacy study examining the antitumour effect of E6/7 siRNA when 


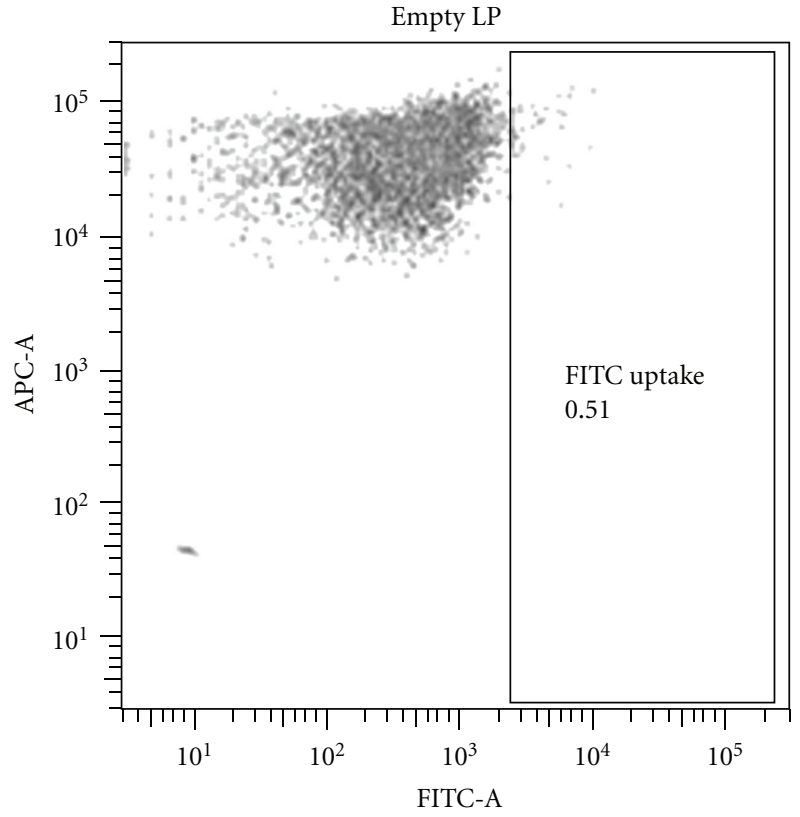

(a)

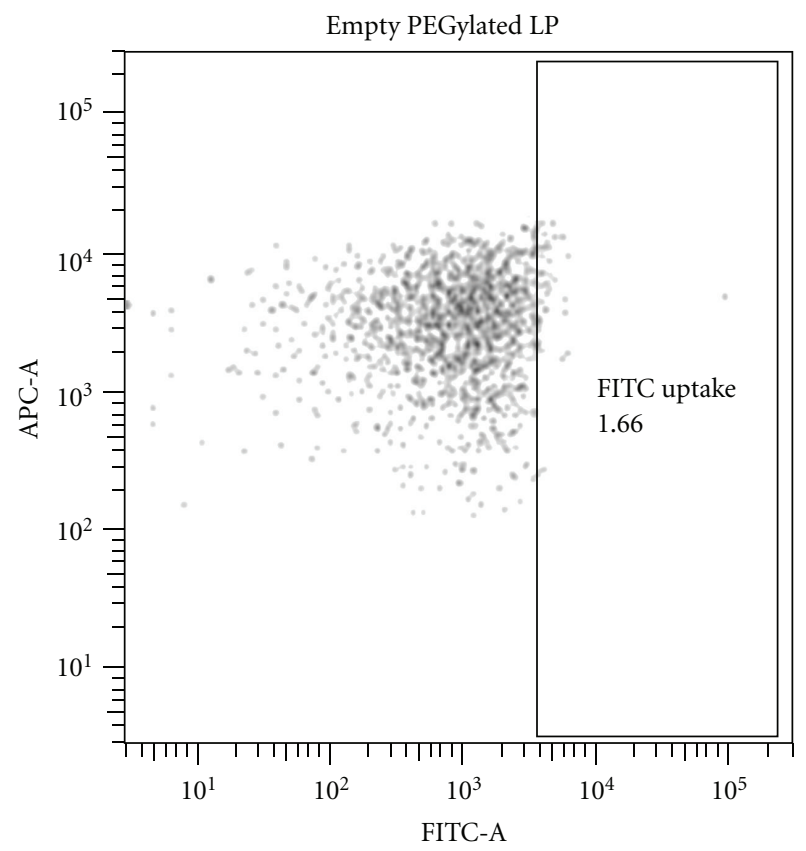

(c)

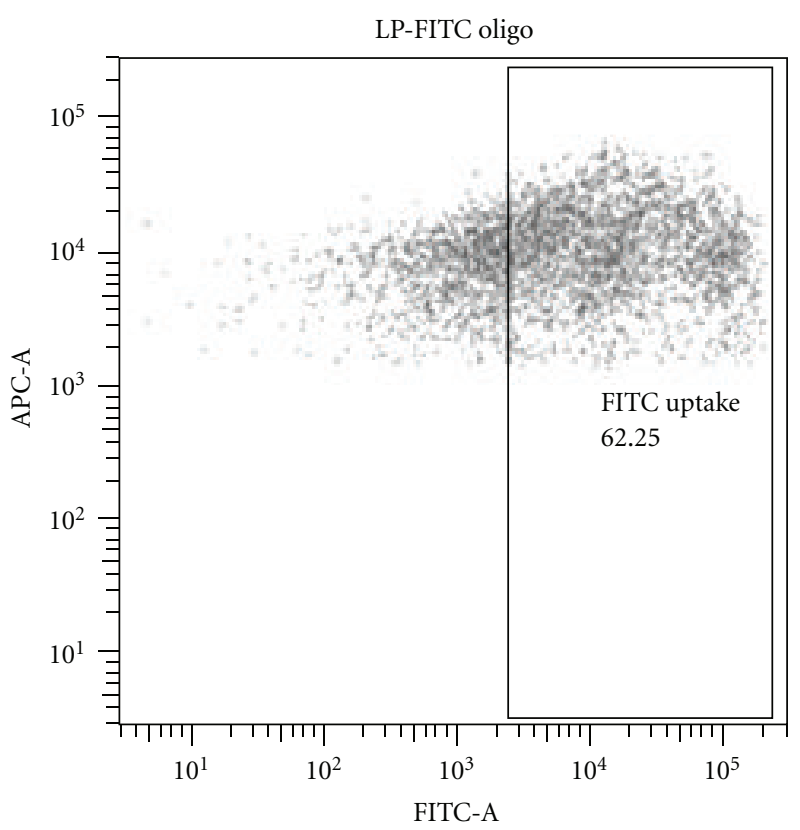

(b)

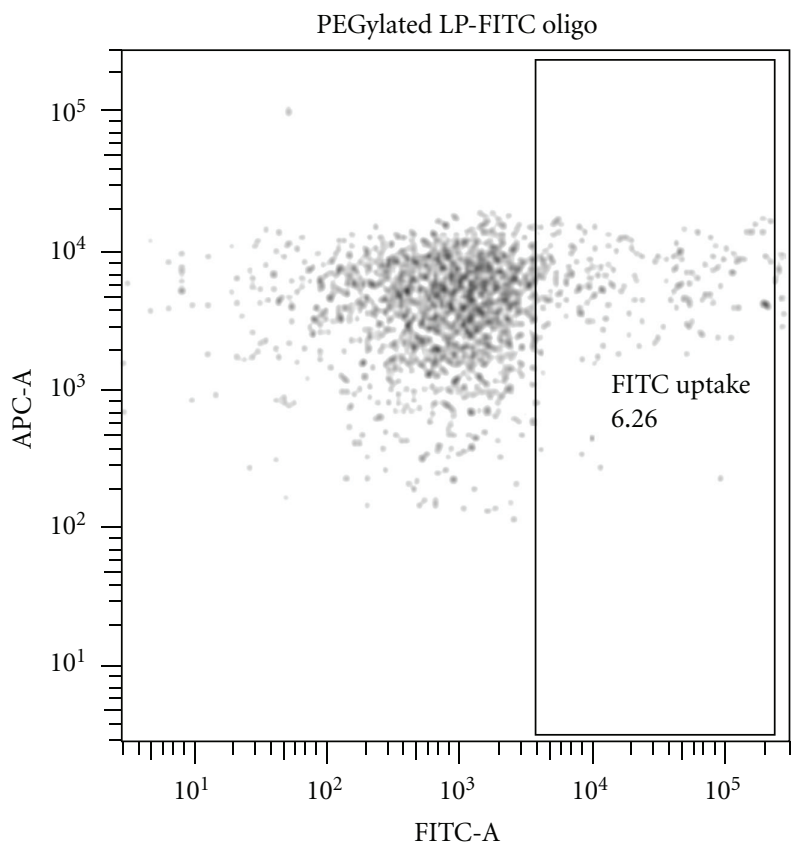

(d)

FIGURE 1: Uptake of FITC-oligonucleotides by intraperitoneal macrophages at 6 hrs after i.p. administration. Oligonucleotides were delivered using either (A) non-PEGylated or (B) PEGylated liposomes (LP), and macrophages were identified via APC-CD11b and PE-F4/80 staining. FACS plots are gated on APC-CD11b- and PE-F4/80-positive cells, and pooled peritoneal fluid of up to 3 mice was examined for each treatment group.

TABLE 1: Quantitative analysis of the distribution of Alexa Fluor 750-conjugated oligonucleotides in tumours and major first-pass organs at $24 \mathrm{hrs}$ following intraperitoneal administration in mice. Oligonucleotides were delivered using either non-PEGylated or PEGylated liposomes (LP). The fluorescent images are presented in Figures 2 and 3.

\begin{tabular}{lcccc}
\hline & \multicolumn{3}{c}{ Average percentage of the total fluorescence intensity detected } \\
& Tumour & Liver & Kidney & Spleen \\
\hline Non-PEGylated LP & $7.5 \%$ & $45.7 \%$ & $15.7 \%$ & $30.0 \%$ \\
PEGylated LP & $65.9 \%$ & $20.6 \%$ & $7.8 \%$ & $5.7 \%$ \\
\hline
\end{tabular}




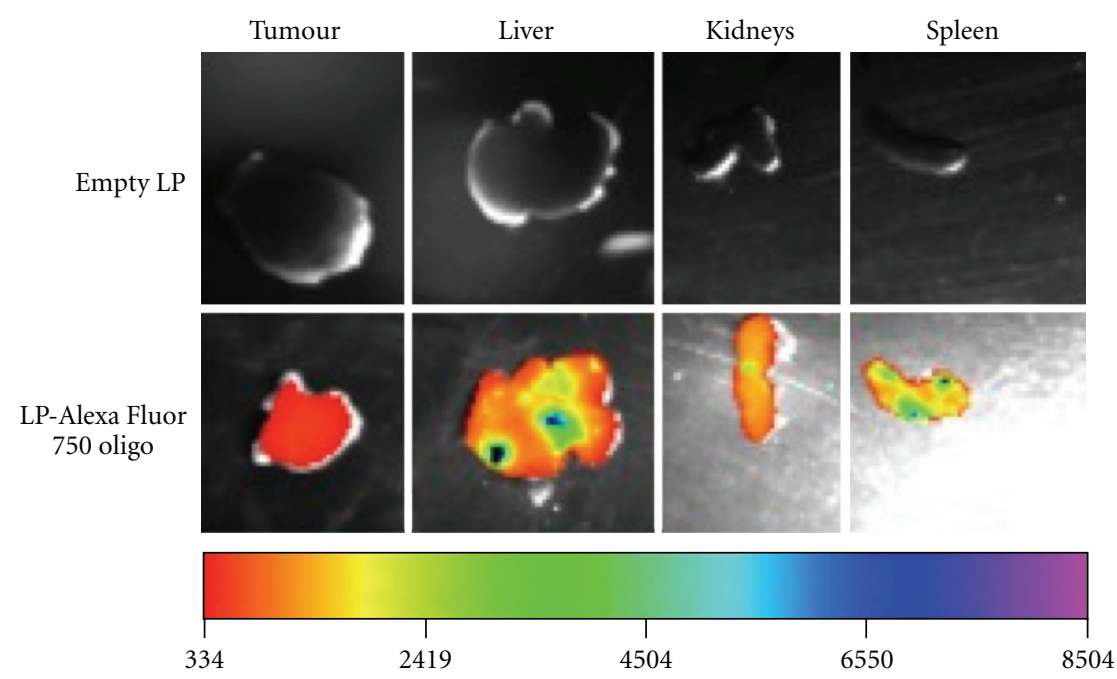

Figure 2: Biodistribution of non-PEGylated liposome-entrapped Alexa Fluor 750-conjugated oligonucleotides in tumours and major firstpass organs at $24 \mathrm{hrs}$ following intraperitoneal (i.p.) administration in mice.

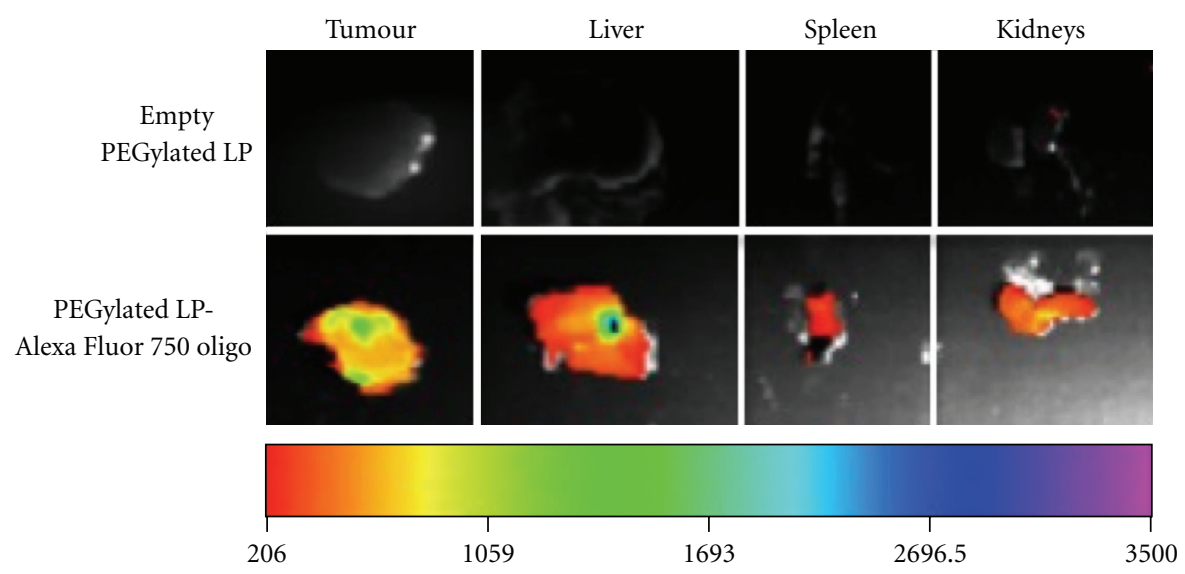

FIGURE 3: Biodistribution of PEGylated liposome-entrapped Alexa Fluor 750-conjugated oligonucleotides in tumours and major first-pass organs at $24 \mathrm{hrs}$ following intraperitoneal (i.p.) administration in mice.

they are delivered intraperitoneally using these PEGylated lipoplexes. E6/7 was chosen as the target oncogene as it is exclusively present in tumour cells of cervical origin and its presence is absolutely essential for the proliferation of TC-1 cells used in this study [20]. Naked E6/7 siRNA, however, would not be able to be taken up by tumour cells readily following systemic administration [8]. Effective tumour delivery of these E6/7-targeted siRNAs using an appropriate carrier was therefore essential in order to achieve a significant reduction in tumour growth rate, as we have previously demonstrated with i.v. route of administration [8]. Indeed, a $45 \%$ decrease in average tumour size was observed in mice treated with 3 doses of i.p. administered PEGylated siE6/7-containing lipoplexes when compared to vehicle-only-treated mice (Figure $4, P<.05$ ). While similar level of tumour size reduction was reported to be achieved with a much more frequent administrations of a much lower dose/volume $(2 \mu \mathrm{g} / 12 \mu \mathrm{L} /$ dose, 12 injections $)$ of siRNA in another study [19], we have found that the administration of such a low volume of lipoplexes into the peritoneal cavity is unlikely to yield a consistent level of delivery to extraperitoneal tumours (data not shown). It must be noted, however, that a slight reduction in tumour size was also observed in nontargeted siRNA or empty liposome treated groups compared to vehicle-only controls, although the level of reduction was not statistically significant (Figure 4). It is speculated at this time that this nonspecific reduction in tumour size observed could have been contributed by the mice's intrinsic response to repeated administrations of large volume/dose of liposomes. It remains to be investigated in the future as to whether enhanced tolerability and less nonspecific effects could occur when the dose was infusing slowly into mice without losing the efficacy [6]. Despite this, our results clearly indicated the promise of PEGylated liposomes in delivering siRNA to extraperitoneal tumours following i.p. administration. 


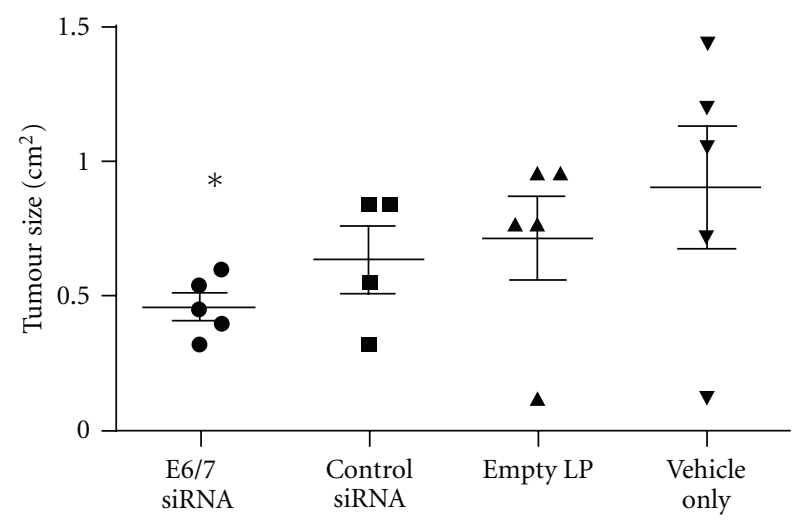

FIGURE 4: Inhibition of extraperitoneal TC-1 tumour growth by i.p. delivered siE6/7-containing PEGylated lipoplexes in mice. Mice received siRNA treatments on days 3, 7, and 10 following tumour cell inoculatios and tumour size was measured using callipers on day 13. All siRNAs were delivered using PEGylated liposomes (LP). Five mice were used per treatment group, and the error bars represent standard deviations. ${ }^{*} P<.05$, significantly different from the vehicle-only treatment group.

\section{Conclusions}

To our knowledge, this is the first paper which systematically investigates the feasibility of delivering siRNA to extraperitoneal tumours following i.p. administration. We showed that, in contrast to non-PEGylated liposomes, PEGylated liposomes were able to facilitate the escape of siRNA from first-pass organs and deliver siRNA efficiently to extraperitoneal tumours after i.p injections. With the incorporation of E6/7-targeted siRNA, significant antitumour effect was observed in mice bearing extraperitoneal TC-1 tumours. Given the inconvenience of repeated i.v. administration along with the ease of aggregate formation for siRNAcontaining formulations, our findings offer an attractive alternative for the treatment of cancers of peritoneal origin with the presence of extraperitoneal metastasis.

\section{Acknowledgments}

S. Wu was supported by National Health and Medical Research Council (NHMRC). This work was supported by grants from the Australian Cancer Research Foundation, NHMRC (Project 455890) and the Cancer Council Queensland. The authors would like to thank Richa Singhania for technical assistance and Kim Woolley and Michelle Kappler for their assistance in animal studies. A. Singhania and S. Wu contributed equally to the work.

\section{References}

[1] C. N. Landen, W. M. Merritt, L. S. Mangala et al., "Intraperitoneal delivery of liposomal siRNA for therapy of advanced ovarian cancer," Cancer Biology and Therapy, vol. 5, no. 12, pp. 1708-1713, 2006.

[2] H. Giladi, M. Ketzinel-Gilad, L. Rivkin, Y. Felig, O. Nussbaum, and E. Galun, "Small interfering RNA inhibits hepatitis B virus replication in mice," Molecular Therapy, vol. 8, no. 5, pp. 769776, 2003.

[3] M. Díaz-Hernández, J. Torres-Peraza, A. Salvatori-Abarca et al., "Full motor recovery despite striatal neuron loss and formation of irreversible amyloid-like inclusions in a conditional mouse model of Huntington's disease," Journal of Neuroscience, vol. 25, no. 42, pp. 9773-9781, 2005.

[4] S. Y. Wu and N. A. J. McMillan, "Lipidic systems for in vivo siRNA delivery," AAPS Journal, vol. 11, no. 4, pp. 639-652, 2009.

[5] M. E. Davis, J. E. Zuckerman, C. H. J. Choi et al., "Evidence of RNAi in humans from systemically administered siRNA via targeted nanoparticles," Nature, vol. 464, no. 7291, pp. 10671070, 2010.

[6] K. Chaudhary, S. Haddadin, R. Nistala, and C. Papageorgio, "Intraperitoneal drug therapy: an advantage," Current Clinical Pharmacology, vol. 5, no. 2, pp. 82-88, 2010.

[7] M. Ghionzoli, M. Cananzi, A. Zani et al., "Amniotic fluid stem cell migration after intraperitoneal injection in pup rats: implication for therapy," Pediatric Surgery International, vol. 26, no. 1, pp. 79-84, 2010.

[8] S. Y. Wu, A. Singhania, M. Burgess et al., "Systemic delivery of E6/7 siRNA using novel lipidic particles and its application with cisplatin in cervical cancer mouse models," Gene Therapy, vol. 18, no. 1, pp. 14-22, 2011.

[9] M. Teo, "Peritoneal-based malignancies and their treatment," Annals of the Academy of Medicine Singapore, vol. 39, no. 1, pp. 54-57, 2010.

[10] D. S. Alberts, P. Y. Liu, E. V. Hannigan et al., "Intraperitoneal cisplatin plus intravenous cyclophosphamide versus intravenous cisplatin plus intravenous cyclophosphamide for stage III ovarian cancer," The New England Journal of Medicine, vol. 335, no. 26, pp. 1950-1955, 1996.

[11] M. Markman, B. N. Bundy, D. S. Alberts et al., "Phase III trial of standard-dose intravenous cisplatin plus paclitaxel versus moderately high-dose carboplatin followed by intravenous paclitaxel and intraperitoneal cisplatin in small-volume stage III ovarian carcinoma: an intergroup study of the gynecologic oncology group, Southwestern Oncology Group, and Eastern Cooperative Oncology Group," Journal of Clinical Oncology, vol. 19, no. 4, pp. 1001-1007, 2001.

[12] D. K. Armstrong, B. Bundy, L. Wenzel et al., "Intraperitoneal cisplatin and paclitaxel in ovarian cancer," The New England Journal of Medicine, vol. 354, no. 1, pp. 34-43, 2006.

[13] R. F. Hwang, E. M. Gordon, W. F. Anderson et al., "Gene therapy for primary and metastatic pancreatic cancer with intraperitoneal retroviral vector bearing the wild-type p53 gene," Surgery, vol. 124, no. 2, pp. 143-151, 1998.

[14] J. Deshane, G. P. Siegal, M. Wang et al., "Transductional efficacy and safety of an intraperitoneally delivered adenovirus encoding an anti-erbB-2 intracellular single-chain antibody for ovarian cancer gene therapy," Gynecologic Oncology, vol. 64, no. 3, pp. 378-385, 1997.

[15] P. Rimessi, P. Sabatelli, M. Fabris et al., "Cationic PMMA nanoparticles bind and deliver antisense oligoribonucleotides allowing restoration of dystrophin expression in the $\mathrm{mdx}$ mouse," Molecular Therapy, vol. 17, no. 5, pp. 820-827, 2009.

[16] J. G. Fewell, M. Matar, J. S. Rice et al., "Treatment of disseminated ovarian cancer using nonviral interleukin-12 gene therapy delivered intraperitoneally," Journal of Gene Medicine, vol. 11, no. 8, pp. 718-728, 2009.

[17] T. J. Yeatman and A. F. Chambers, "Osteopontin and colon cancer progression," Clinical and Experimental Metastasis, vol. 20, no. 1, pp. 85-90, 2003. 
[18] S. Yachida, S. Jones, I. Bozic et al., "Distant metastasis occurs late during the genetic evolution of pancreatic cancer," Nature, vol. 467, no. 7319, pp. 1114-1117, 2010.

[19] X. Y. Niu, Z. L. Peng, W. Q. Duan, H. Wang, and P. Wang, "Inhibition of HPV 16 E6 oncogene expression by RNA interference in vitro and in vivo," International Journal of Gynecological Cancer, vol. 16, no. 2, pp. 743-751, 2006.

[20] K. Y. Lin, F. G. Guarnieri, K. F. Staveley-O'Carroll et al., "Treatment of established tumors with a novel vaccine that enhances major histocompatibility class II presentation of tumor antigen," Cancer Research, vol. 56, no. 1, pp. 21-26, 1996.

[21] C. H. Lee, Y. H. Ni, C. C. Chen, C. K. Chou, and F. H. Chang, "Synergistic effect of polyethylenimine and cationic liposomes in nucleic acid delivery to human cancer cells," Biochimica et Biophysica Acta, vol. 1611, no. 1-2, pp. 55-62, 2003.

[22] S. Y. Wu, L. N. Putral, M. Liang, H. I. Chang, N. M. Davies, and N. A. J. McMillan, "Development of a novel method for formulating stable sirna-loaded lipid particles for in vivo use," Pharmaceutical Research, vol. 26, no. 3, pp. 512-522, 2009.

[23] B. Romberg, W. E. Hennink, and G. Storm, "Sheddable coatings for long-circulating nanoparticles," Pharmaceutical Research, vol. 25, no. 1, pp. 55-71, 2008.

[24] S. A. Johnstone, D. Masin, L. Mayer, and M. B. Bally, "Surfaceassociated serum proteins inhibit the uptake of phosphatidylserine and poly(ethylene glycol) liposomes by mouse macrophages," Biochimica et Biophysica Acta, vol. 1513, no. 1, pp. 25-37, 2001.

[25] P. H. Sugarbaker, O. A. Stuart, and C. P. Carmignani, "Pharmacokinetic changes induced by the volume of chemotherapy solution in patients treated with hyperthermic intraperitoneal mitomycin C," Cancer Chemotherapy and Pharmacology, vol. 57, no. 5, pp. 703-708, 2006.

[26] R. L. Dedrick, C. E. Myers, P. M. Bungay, and V. T. Devita Jr., "Pharmacokinetic rationale for peritoneal drug administration in the treatment of ovarian cancer," Cancer Treatment Reports, vol. 62, no. 1, pp. 1-11, 1978.

[27] A. Rippe, C. Rippe, K. Swärd, and B. Rippe, "Disproportionally low clearance of macromolecules from the plasma to the peritoneal cavity in a mouse model of peritoneal dialysis," Nephrology Dialysis Transplantation, vol. 22, no. 1, pp. 88-95, 2007.

[28] P. Keshaviah, P. F. Emerson, E. F. Vonesh, and J. C. Brandes, "Relationship between body size, fill volume, and mass transfer area coefficient in peritoneal dialysis," Journal of the American Society of Nephrology, vol. 4, no. 10, pp. 1820-1826, 1994.

[29] D. S. Kohane, J. Y. Tse, Y. Yeo, R. Padera, M. Shubina, and R. Langer, "Biodegradable polymeric microspheres and nanospheres for drug delivery in the peritoneum," Journal of Biomedical Materials Research Part A, vol. 77, no. 2, pp. 351361, 2006.

[30] B. Roser, “The migration of macrophages in vivo," in Mononuclear Phagocytes, R. van Furth, Ed., pp. 166-174, Blackwell Scientific Publications, Oxford, UK, 1970.

[31] G. Lukas, S. D. Brindle, and P. Greengard, "The route of absorption of intraperitoneally administered compounds," Journal of Pharmacology and Experimental Therapeutics, vol. 178, no. 3, pp. 562-564, 1971.

[32] M. F. Abu-Hijleh, O. A. Habbal, and S. T. Moqattash, "The role of the diaphragm in lymphatic absorption from the peritoneal cavity," Journal of Anatomy, vol. 186, no. 3, pp. 453-467, 1995.

[33] B. Rippe, "A three-pore model of peritoneal transport," Peritoneal Dialysis International, vol. 13, no. 2, pp. S35-S38, 1993. 

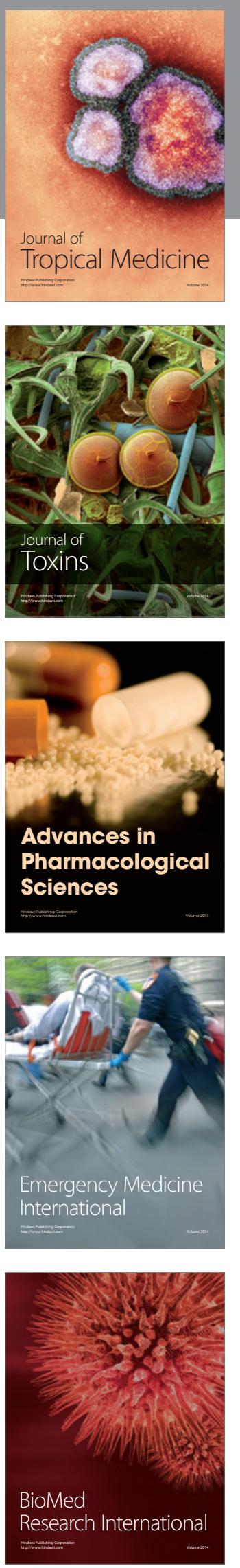
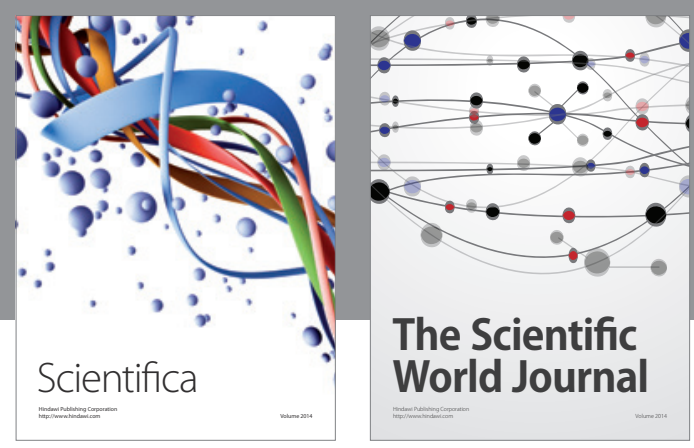

The Scientific World Journal
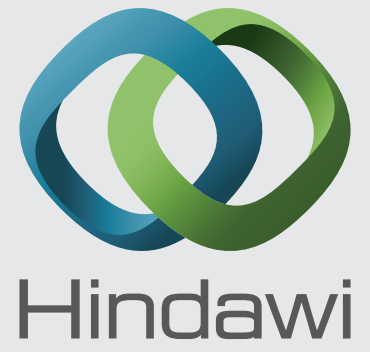

Submit your manuscripts at

http://www.hindawi.com
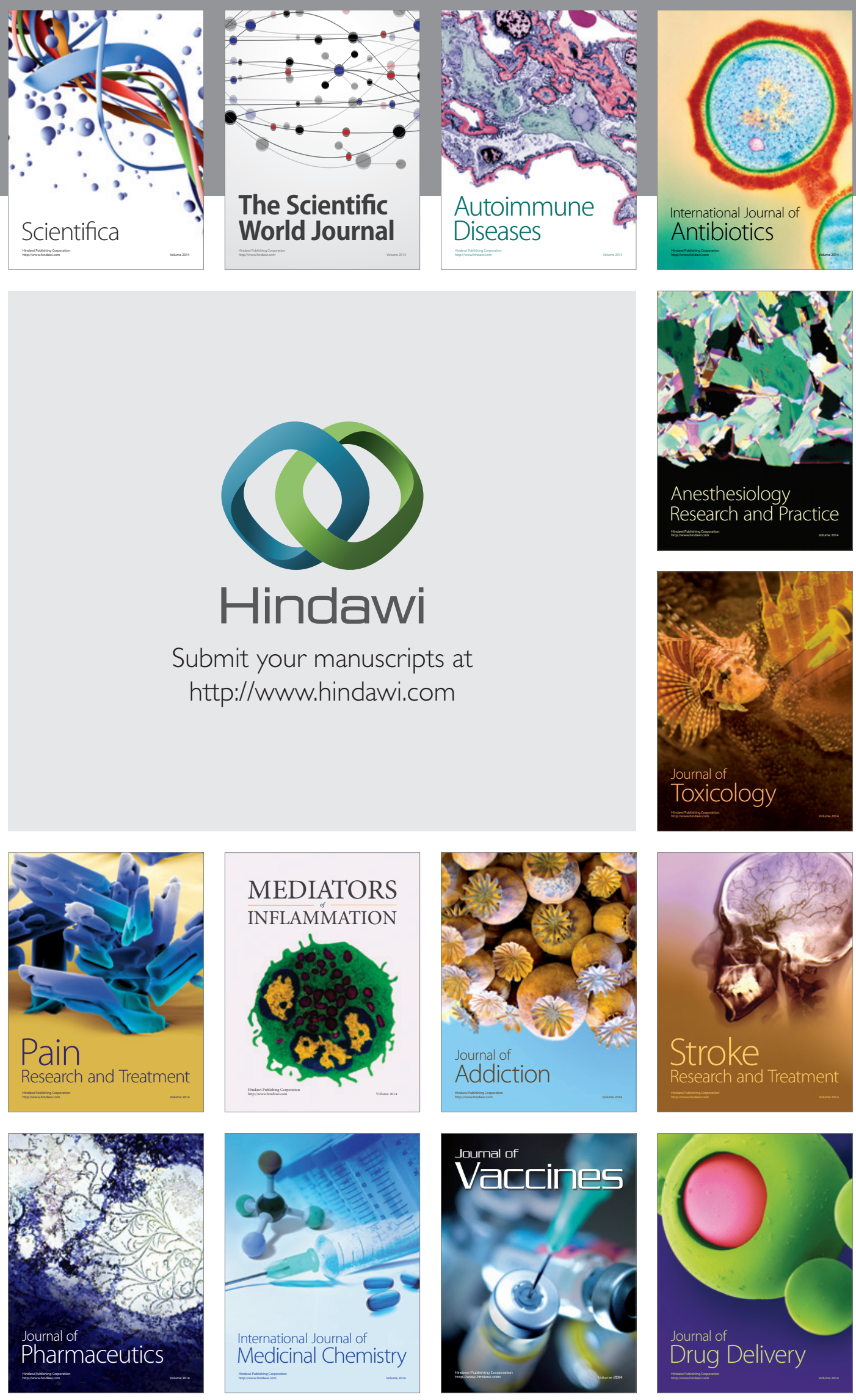